\title{
Lugano Lymphoma Response Classification Complete Remission by PET
}

National Cancer Institute

\section{Source}

National Cancer Institute. Lugano Lymphoma Response Classification Complete

Remission by PET. NCI Thesaurus. Code C142795.

A score of 1, 2, or 3 on a 5-point PET scale in nodal or extranodal sites with or without a residual mass. No evidence of FDG-avid disease in the marrow. 